\title{
Integration of Quantum Key Distribution in Metropolitan Area Networks
}

\author{
A. Poppe, ${ }^{1 *}$ B. Schrenk, ${ }^{1}$ F. Hipp, ${ }^{1}$ M. Peev,${ }^{1}$ S. Aleksic, ${ }^{2}$ G. Franzl, ${ }^{2}$ A. Ciurana, ${ }^{3}$ V. Martin ${ }^{3}$ \\ 1 Optical Quantum Technology, Safety \& Security Department, AIT Austrian Institute of Technology GmbH, \\ Donau-City-Strasse 1, 1220 Vienna, Austria \\ 2 Institute of Telecommunications, Vienna University of Technology, \\ Favoritenstrasse 9-11/E389, 1040 Vienna, Austria \\ 3 Research Group on Quantum Information and Computation, Universidad Politécnica de Madrid, Spain \\ Corresponding author: andreas.poppe@ait.ac.at
}

\begin{abstract}
The deployment of Quantum Key Distribution forces the development of QKD-links to be operated in current and next-generation photonic metro-access networks. These highly heterogeneous architectures determine the conditions QKD-links need to be optimized for. OCIS codes: (060.5565) Fiber optics and optical communications: Quantum communications; (270.5568) Quantum optics: Quantum cryptography; $(060.4265)$ Fiber optics and optical communications: Networks, wavelength routing.
\end{abstract}

\section{Introduction and motivation}

Quantum Key Distribution (QKD) is maturing quickly. The performance of recently developed systems steadily approaches physical and (current and near future) technological limits. Unfortunately, these are all adapted and optimized to point-to-point operation and require a dark fibre, which makes them unsuited for immediate integration standard communication systems on an existing infrastructure. For this reason cost of ownership (dark fibre lease) is very high and for a year tends to exceed the purchase costs. This is main factor that hinders a wide deployment and restricts QKD application to a niche market. This leads to the production of a very small number of devices, which in turn implies a significant overhead due to development costs and high production expenses. In principle, direct dark fibers would be ideally suited to connect a small number of users. Moreover, the usage of the needed fibers could be optimized by installing a dedicated infrastructure of trusted repeater stations (nodes) as vertices in a graph, the edges being the direct quantum channels [1]. Unfortunately even in the case of lower costs for higher number of produced devices the rent of the fiber infrastructure remains the dominant factor. In a nutshell: Even though present day QKD offers unprecedented degree of security and sufficient engineering, it comes at a forbiddingly high price mainly due to the infrastructure compatibility. The technology cannot hope for broader proliferation until it can be adapted to fit in existing and future communication networks.

As a first step, a dual use for classical and quantum communications has been shown recently [2,3]. A strategy for better integration in communication infrastructure calls for the development of a QKD system that is adapted by design to classical networks and in particular allows for using standard communication lines as a quantum channel. Here we assume the optical communication to be standard and not modifiable in the case of introduction of additional QKD systems.

\section{Synthesis scenario for photonic metro-access networks with integrated QKD}

The deployment scenario we want to address here facilitated key distribution among arbitrary users in a metropolitan area. The synthesis to an integrated QKD network is carried out in a way that existing fiber infrastructure is reused while additional telecom-grade elements are introduced at the network nodes. Figure 1 shows the principal reference architecture of a typical metro-access network where a metro DWDM ring collects traffic from the access trees. Down- and upstream aggregation in the metro add/drop (A/D) node occurs through remote protocol termination at the access-side optical line terminals (OLT) and the DWDM transceivers that are connected to the reconfigurable add/drop multiplexer (ROADM) of the double ring-based metro network. In this way the traffic arising locally at the slave central office $(\mathrm{CO})$ can be effectively backhauled to a centralized master$\mathrm{CO}$. Although recent schemes that address metro-convergence propose to avoid optic/electric/optic conversion in order to achieve active site consolidation by means of remote amplification and colourless optical network terminals (ONT) at the user premises [4], the chosen case is closer to brown-field architectures deployed nowadays. Passive splitters between the feeder and the drop segment of the access network, which are laid out as either power-splitting or wavelength multiplexing device according to the respective PON architecture, connect the classic+QKD customer premises equipment (CPE) of multiple users to the respective OLT. 


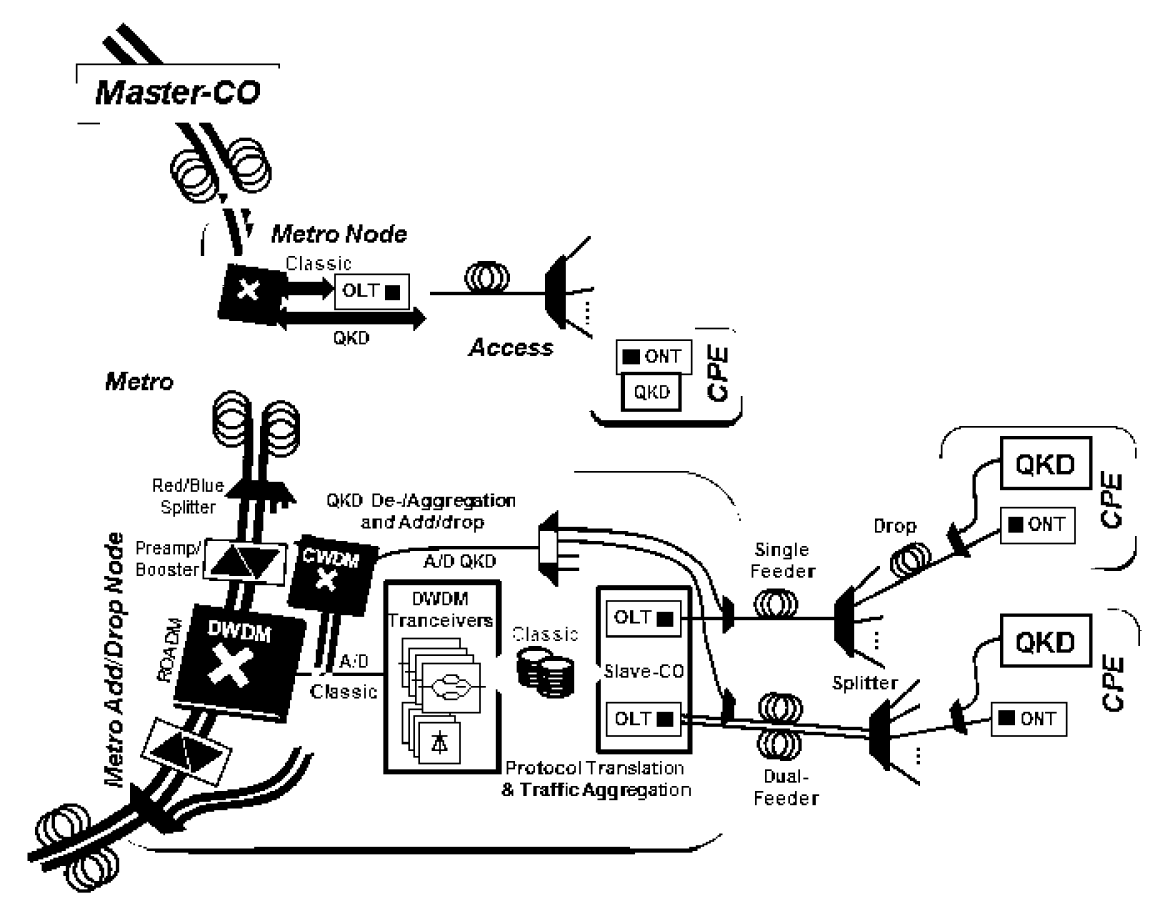

Fig.1. Principal architecture of a typical metro-access network with additional building blocks to enable operation of QKD-links over the network

Different passive optical network (PON) standards exist for the access segment, all of them laid out without looking into co-existence issues concerning QKD systems, e.g. through reserving remote wavebands with wide spectral guardband for the by far more vulnerable quantum channels. Table 1 confirms that most of these standards span across the available fiber spectrum in the $\mathrm{O}-$ and $\mathrm{S} / \mathrm{C} / \mathrm{L}$-bands, either in downstream or in upstream direction. In Table 1 the line rate as well as the specified minimum and maximum transmitter (TX) power is included.

\begin{tabular}{|c|c|c|c|c|c|c|c|c|c|}
\hline & & PtP GbE & $\begin{array}{c}\text { PtP 10 } \\
\text { GbE }\end{array}$ & EPON & GPON & $\begin{array}{c}10 G \\
\text { EPON }\end{array}$ & XG PON & WDM PON & \begin{tabular}{|c} 
WDM-TDM \\
PON
\end{tabular} \\
\hline \multirow{3}{*}{ 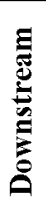 } & $\begin{array}{c}\text { Wavelength [n } \\
\text { m] }\end{array}$ & 1550 & 1330 & $\begin{array}{c}1490, \\
1550 \text { (video) }\end{array}$ & \begin{tabular}{c|}
1490, \\
1550 (video)
\end{tabular} & 1577 & 1577 & $1548-1577$ & $1548-1577$ \\
\hline & $\begin{array}{c}\text { Line rate } \\
\text { [Gbps] }\end{array}$ & 1.25 & 10.3125 & 1.25 & 2.488 & 10.3125 & 9.953 & 10.3125 & 10.3125 \\
\hline & $\begin{array}{l}\text { TX power } \\
\text { [dBm] }\end{array}$ & $-8, . . .-2$ & $-5, . .2$ & $2.5, . . .7$ & $3, . ., 7$ & $2, . ., 6$ & $2, . ., 6$ & $2, . ., 5$ & $2, . ., 5$ \\
\hline \multirow{3}{*}{ 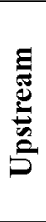 } & $\begin{array}{c}\text { Wavelength[n } \\
\mathrm{m}]\end{array}$ & 1310 & 1270 & 1310 & 1310 & 1270 & 1270 & $1520-1547$ & $1520-1547$ \\
\hline & $\begin{array}{c}\text { Line rate } \\
\text { [Gbps] }\end{array}$ & 1.25 & 10.3125 & 1.25 & 1.244 & 1.25 & 2.488 & 1.25 & 1.25 \\
\hline & $\begin{array}{c}\text { TX power } \\
\text { [dBm] }\end{array}$ & $-8, . .,-2$ & $-5, . ., 2$ & $-1, . ., 4$ & $0.5, . ., 5$ & $2, . ., 6$ & $2, . ., 6$ & $1, . ., 4$ & $1, . ., 4$ \\
\hline
\end{tabular}

Table. 1. Overview of PON standards and other legacy systems found in optical access networks. For downstream (DS) and upstream (US) direction the center wavelength, specified line rates and the range of launched power is indicated.

\section{QKD-links in metro-access networks}

A typical scenario for the integration of QKD channels of deployment in metro-access architectures is shown depicted in Fig. 1. Here The connectivity model for QKD is built on a we assume a direct end-to-end connection of QKD-links connecting between a pairs of users. These connections are provided as virtual point-to-point connections over the existing DWDM metro and WDM or TDM or WDM/TDM access segments and can be flexibly established through ROADM technology along the metro ring.

The nonlinear effect of Raman scattering causes fiber-based optical transmission lines to produce out-of-band noise photons that are spectrally displaced by tens to hundreds of nanometers from the central wavelength [3]. In 
contrast to other classical communication channels on the same fiber that are not disturbed, QKD-links are flooded by uncorrelated noise photons resulting in highly unfavorable signal-to-crosstalk ratios.

The amount of noise photons depends on many parameters. First of all, larger magnitudes are observed at lower photon-energies (i.e. higher wavelength, Stoke-radiation) as for generated photons with lower wavelength (i.e. antiStoke-radiation). Depending on the deployed PON standard (Table 1), more or less wavelength bands (e.g., CWDM channels) become unfavorable for QKD. Especially those splitting up- and downstream in two remote wavebands at $1310 \mathrm{~nm}$ and $1550 \mathrm{~nm}$ (e.g. GPON or XG-PON) impose severe challenges to maintain a QKD channel. On the other hand, standards that multiplex full-duplex channels in the same waveband (e.g. WDM-PON) leave an untouched spectral window for the establishment of QKD links. Both the transmitter power and its center wavelength have a strong influence on the level of background noise in the QKD channel.

We calculated the worst case with respect to the launched power with a simultaneous selection of the wavelength within the specified band for different scenarios. A typical plot for GPON and XG-PON is shown in Figure 2. To yield the lowest amount of noise photons the QKD-link embedded in a GPON should be operated below 1300nm and be independent of the operation direction (upstream or downstream). For the XG-PON the optimal wavelength of operation would be close to the $\mathrm{OH}$-absorption line around $1400 \mathrm{~nm}$. In contrary, noise arising from the amplified DWDM link at the metro ring shows a $10 \mathrm{x}$ lower noise background as the Raman seed in confined in the C-band.

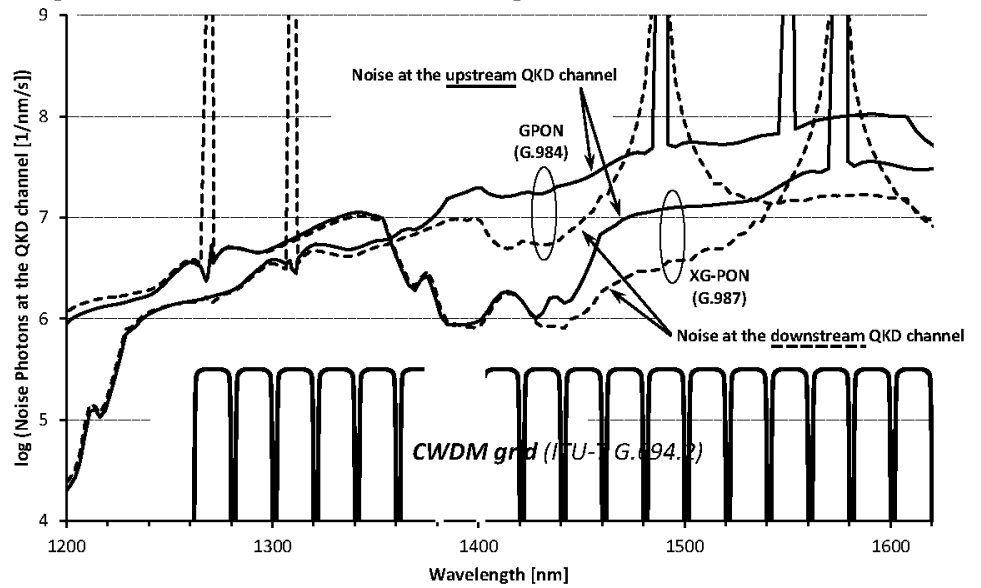

Fig. 2. Noise introduced in the up-/downstream QKD channel arising from GPON and XG-PON access network standards deployed over a single-feeder access distribution network, including also video overlay.

As clearly seen in Figure 2 the direction of operation of the QKD-link can be critical. This is the case when the plots of the generated noise in upstream and downstream directions are different. To the end, the qubits sent from a QKDdevice should not co-propagate with the telecom signal at a small wavelength difference. In counter-propagation, the backwards radiation could still be pronounced enough to prevent a typical QKD-link from generating secure keys.

\section{Conclusions and Outlook}

Different deployment scenarios of QKD-links in metro-access networks need to be addressed in future by taking the amount of highly detrimental noise photons generated by Raman radiation into account. The noise background depends strongly on the type of the access network standard that co-exist with the QKD links in the field. Other deployment scenarios as to serve many users over a $\mathrm{CO}$ or to use entangled photon pairs are a reasonable next step.

Acknowledgement: This work was funded by the Austrian Research Promotion Agency FFG under contract no. 835926 (QKD-TELCO).

\section{References}

[1] M. Peev et al., "The SECOQC quantum key distribution network in Vienna," N. J. Phys. 11, 075001 (2009).

[2] K. A. Patel, J. F. Dynes, I. Choi, A. W. Sharpe, A. R. Dixon, Z. L. Yuan, R. V. Penty, and A. J. Shields, "Coexistence of High-Bit-Rate Quantum Key Distribution and Data on Optical Fiber," Phys. Rev. X 2, 041010 (2012).

[3] P Eraerds, N Walenta, M Legré, N Gisin, and H Zbinden, "Quantum key distribution and 1 Gbps data encryption over a single fibre," N. J. Phys. 12, 063027 (2010).

[4] B. Schrenk et al., "Demonstration of a Remotely Dual-Pumped Long-Reach PON for Flexible Deployment," IEEE/OSA J. Lightwave Technol. 30, 953 (2012).

[5] A. Ciurana, J. Martinez-Mateo, M. Peev, A. Poppe, N. Walenta, H. Zbinden, and V. Martin, "Quantum metropolitan optical network based on wavelength division multiplexing," accepted for publication in Optics Express.

[6] S. Gringeri et al., "Flexible Architectures for Optical Transport Nodes and Networks," IEEE Communications Magazine, Jul. 2010, 40, (2010). 